\title{
The Adaptive Approach to Measurements of Deviations of Cylindrical Parts
}

\author{
Stanisław Adamczak ${ }^{1}$, Dariusz Janecki ${ }^{2}$, Krzysztof Stępień ${ }^{3, *}$ \\ Kielce University of Technology Faculty of Mechatronics and Mechanical Engineering, Address: Al. 1000-lecia P. P. 7, 25-314 Kielce, Poland \\ Kielce University of Technology Faculty of Mechatronics and Mechanical Engineering, Address: Al. 1000-lecia P. P. 7, 25-314 Kielce, Poland \\ Kielce University of Technology Faculty of Mechatronics and Mechanical Engineering, Address: Al. 1000-lecia P. P. 7, 25-314 Kielce, Poland
}

\begin{abstract}
Measurements of form deviations of 3D elements can be conducted with various strategies. Such strategies differ in a number and distribution of sampling points located on an investigated surface. The low number of sampling points can bring about the situation that some surface irregularities cannot be detected by a measuring system. An application of higher density of sampling points, in turn, results in significant lengthening of measurement time, which is undesirable. This is why the authors propose to develop a novel adaptive measurement strategy. Proposed adaptive strategy consists of two stages: a preliminary measurement and additional measurements. During the preliminary measurement, an investigated area is scanned along a preselected trajectory. If the measurement results show that there is a significant change in sensor readings in a certain fragment of an investigated surface, then we conduct additional measurements in the area where the large change in sensor readings occurred. The paper presents the state-of-the-art on contemporary measurement strategies of 3D elements and fundamentals of the adaptive strategy proposed by the authors.
\end{abstract}

Keywords: measurements of geometrical quantities; measurement strategy; form errors

\section{Introduction}

Rotary elements constitute a very significant and numerous group of machine parts. Such machine parts are used in various branches of engineering industry (for example in bearing, automotive or power industry). Such parts are usually cylindrical, spherical, barrel- and saddle-shaped or conical ones. Rotating elements should usually be of very high accuracy. Therefore, it is very important to apply a reliable method of measurements of their form deviations. Usually, the control of the form deviations of rotary parts is based on an analysis of results of 2D measurements. In other words, the control is performed in the following way: measurements of roundness deviations are conducted in a few preselected cross-sections of the part.

It is less common to measure the 3D parameters of the rotary parts. In industrial practice, measurements of 3D parameters are practically limited to an evaluation of outof-cylindricity. Cylindricity measurements are usually performed with special-purpose radial systems. Typical example of such systems is series of Talyrond instruments by Taylor Hobson. Nowadays, radial systems, also called radius-change ones, can be used to measure roundness and cylindricity deviations. In addition, some radius-change instruments can also be used to measure the flatness of the cylinder face. Radius-change instruments are characterized by high accuracy; however, their area of applications is limited, as they usually can be used to measure roundness, cylindricity, or flatness of the face, as it was mentioned above.

Today, in the area of metrology of geometrical quantities, the dynamic develop- 
ment of coordinate metrology can be observed. It contributes to increasing the measurement accuracy of coordinate measuring machines. This is why such type of machines are becoming more and more common to measure form deviations, if tolerances of the part are significantly higher than the maximum permissible error of the machine.

Considering the problem of measurements of 3D parameters of rotary parts, it is noteworthy that it is very important to apply proper measurement strategy. Term "measurement strategy" denotes here distribution of sampling points on the surface under investigation. Thus, this term is closely related to the path along which the sensor moves on the surface to be measured. It is obvious that the applied measurement strategy should allow dense coverage of the investigated surface with a grid of sampling points. On the other hand, it should be noted that the higher the number of sampling points, the longer the measurement time. Therefore, sometimes selecting the proper measurement strategy is not an easy task.

\section{State-of-the-art in measuring strategies for 3D features}

Measurement strategies of 3D parameters described in the scientific literature usually relate to measurements of cylindrical workpieces. In general, they can be divided into three main groups: uniform sampling strategies, strategies fitted to measured surfaces on the basis of predicted pattern of irregularities and so-called adaptive strategies (see Fig. 1).

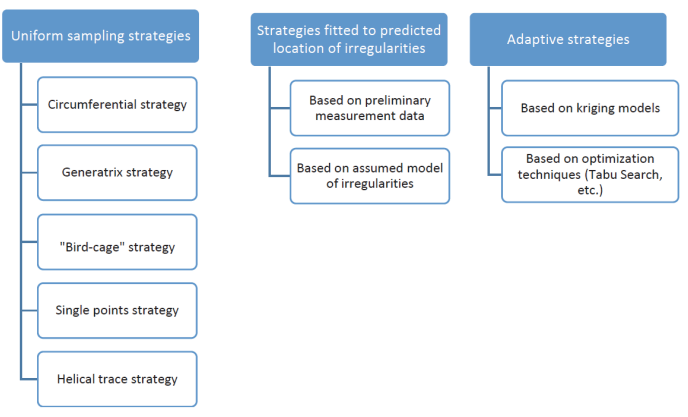

Figure 1: Classification of measurement strategies used to measure form deviations of rotary parts.

Uniform sampling strategies are most common in industrial practice. Usually measurements of form deviations of cylindrical parts are performed with the use of the circumferential and the generatrix strategy that are presented in Fig. 2. a)

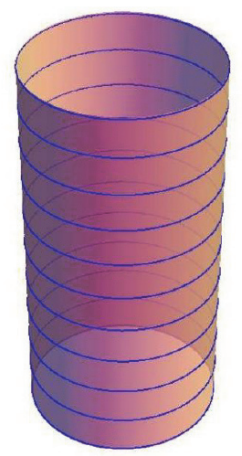

b)

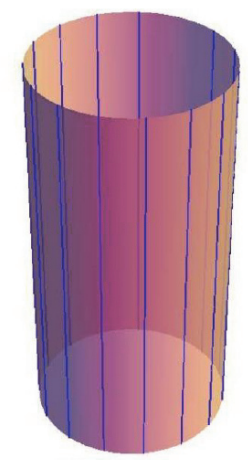

Figure 2: Most common uniform sampling strategies used to measure rotary parts: a) circumferential strategy, b) generatrix strategy.

The strategy that allows obtaining more accurate information about investigated surfaces is the so-called "bird-cage" strategy. This strategy is simply a combination of measurements in cross- and longitudinal sections (see Fig. 3).
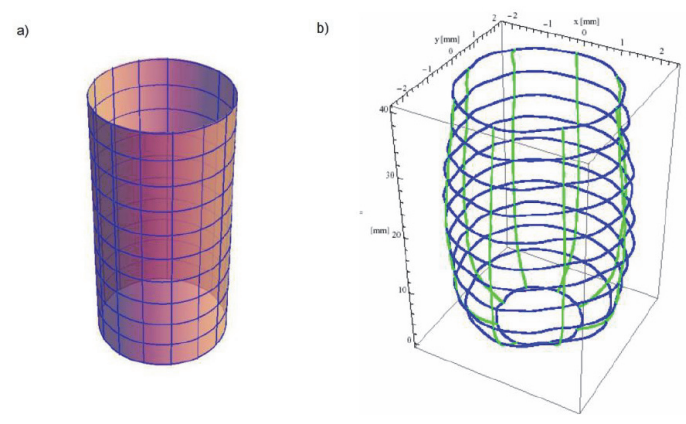

Figure 3: "Bird-cage" strategy: a) the scanning trajectory, b) an example of the application of the strategy in practice.

Due to computational problems, the "bird-cage" strategy has not been available in the software of measuring systems for a long time. At the moment, there are some measurement systems that allow the application of this strategy. The most important advantage of the "bird-cage" strategy is that it allows dense covering of measured surface with a grid of sampling points. Therefore, such results are most reliable. The major drawback of the "bird-cage" strategy is that it is very time consuming.

Single points strategy is less common to mea- 
sure form deviations of cylindrical parts. The reason is that low number of sampling points does not allow obtaining accurate information about investigated surface.

The strategy that was not described in the ISO 12180 standard is the helical trace strategy. This strategy is a compromise solution between the strategies of cross-sections and longitudinal sections and it is quite often applied under industrial conditions. Strategies based on a single points and on helical trace are shown in Fig. 4. a)

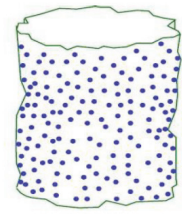

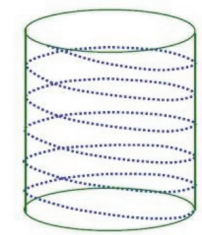

Figure 4: Single points strategy (Fig. 4a) and helical trace strategy (Fig 4b) [1].

In general, it should be noted that the strategies shown in Fig. 2-4 allow uniform sampling. This means that the whole surface of the specimen is scanned in the same way. Thus, for the case of circumferential, generatrix and "bird-cage" strategy all the linear distances between all cross-sections and angular distances between longitudinal sections are the same. The helical trace strategy can also be regarded as a uniform sampling strategy since the parameter that describes the sampling density, which is the pitch angle, remains constant during the measurement. Application of the single points strategies also requires approximate uniform distribution of sampling points on the surface under investigation. It can be performed with the use of specific numerical methods, for example by applying the Hammersley sequence [2].

Uniform sampling strategies are very useful if the surfaces under study are characterized by a regular pattern of form errors. However, significant irregularities are sometimes located only in a certain area of the surface. Such an area should be then sampled with the use of many more points than other areas of the surface. This is the reason why research activities are conducted that are focused on development of a strategy that would denser sampling in areas where significant surface defects are predicted.

Strategies based on predicted location of irregu- larities are fitted to the surface under study on the basis of predicted distribution of surface irregularities. If information about the machining process of the surface is available, then one can predict the most probable location of the irregularities of the surface. For example, cylindrical surfaces of workpieces machined by turning that were placed in a three-jaw chucks are very often characterized by a triangular error (i.e., the third harmonic component is dominant in circumferential profile). Additionally, workpieces that were placed in lathe centers are usually characterized by a saddle-shaped error.

Strategies fitted to predicted models of irregularities can be designed on the basis of data of preliminary measurements or on the basis of assumed pattern of form errors. An example of the former approach is a method of harmonics fitting described in work [3]. This method applies statistical evaluation of characteristics of individual Fourier components of the profile in order to establish as small set of sampling points data as possible that allows reliable evaluation of form errors of the surface under study. The latter approach is given in work [4]. In this method, a preliminary model of the shape of the surface is assumed. The model is described mathematically by the linear combination of the set of base functions (for example, polynomials, Fourier components or eigenfunctions). The model is then used to design the grid of sampling points. After measurement, on the basis of the values in preselected sampling points, the coefficients of assumed model of the linear combination are calculated (taking into account uncertainty of fitting). The coefficients are then used to reconstruct the image of the whole surface under study.

Adaptive strategies, described in the scientific literature, are iterative. In the case of application of such types of strategies, the sampling is conducted in a few stages. The first stage is a preliminary measurement. The preliminary measurement is usually performed with the use of uniform sampling. Next, on the basis of the measured data obtained, a relevant algorithm is applied to find the areas where the risk of occurring of large local surface irregularities is high. After conducting measurements in these areas, the value of presumed coefficient is calculated. The value of this coefficient indicates whether the measurements should be stopped or if it is necessary to conduct the next series of measurements applying denser sampling. Such approach uses 
so-called kriging models quite often. The name of the method comes from the name South African engineer, Krige, who was the first researcher to apply this method to predict changes of the signal in geostatics in the 1960s of the XX. century. At present, these models are increasingly common to predict the location of subsequent sampling points. In kriging models, the signal is modified by using the set of base functions and their coefficients supplemented by a random component whose expected value is equal to zero and for whom the covariance between input and output remains constant [5].

Apart from the application of kriging models, the localization of points, where the profile should be sampled can be conducted with the use of such optimization techniques as Tabu Search, Hybrid Search or coordinate search [6].

\section{The approach to adaptive measurement proposed by the authors}

As was shown in the previous section, measurements of form deviations of 3D elements can be conducted with the use of various strategies, differing in the number and distribution of sampling points located on an investigated surface. A low number of sampling points or measured sections can lead to the situation that some surface irregularities are not detected by a measuring system. An application of higher density of sampling points, in turn, results in significant lengthening of measurement time, which is undesirable, if one takes into account requirements of modern manufacturing processes.

This is the reason why authors have taken efforts aiming at development a novel, adaptive measurement strategy, different from those described in the previous section. Proposed adaptive strategy consists of two stages: a preliminary measurement and additional measurement (one of many - it depends on the results of the signal analysis). The general concept of the strategy is shown in Fig. 5.

During the preliminary measurement, an investigated area is scanned along preselected trajectory. The authors propose to apply three strategies of preliminary scanning: along the helical trace, in preselected cross-sections and in preselected longitudinal sections.

The approach to the evaluation of form deviations of 3D parameters of rotary elements shown in Fig. 5 requires solving some specific problems. One

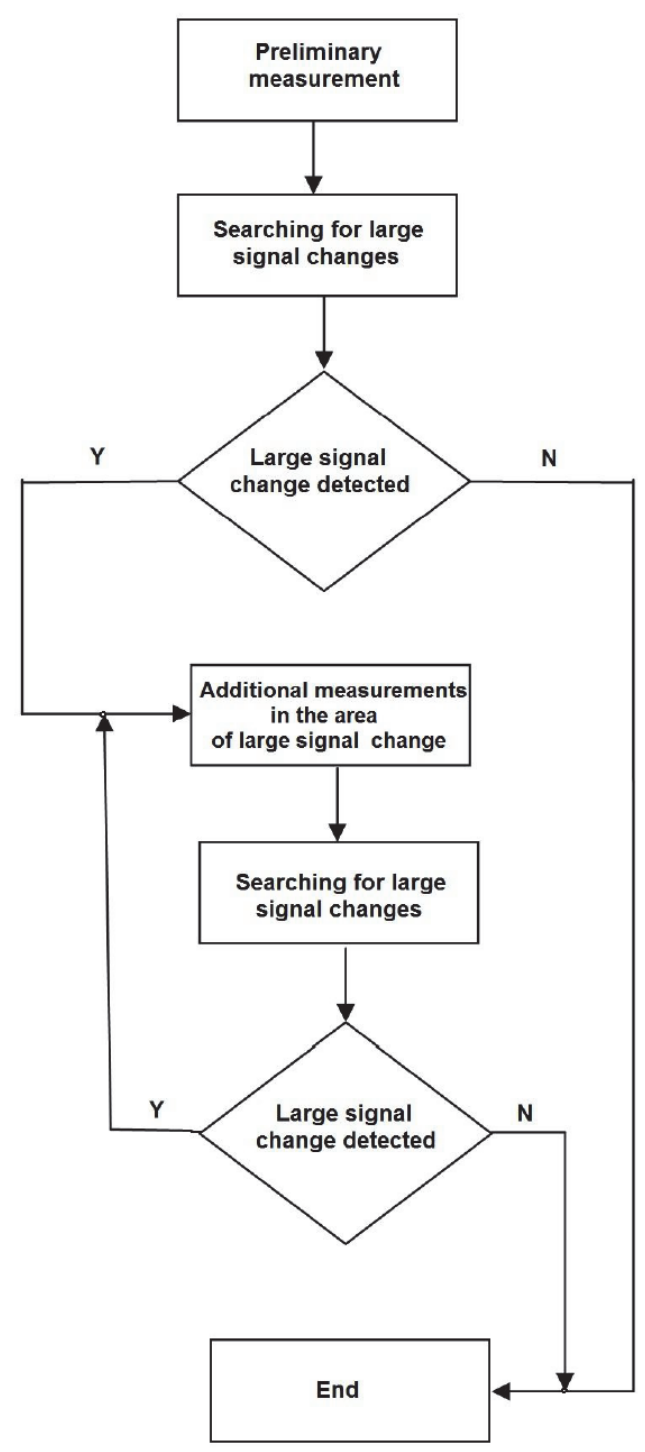

Figure 5: General concept of the adaptive strategy proposed by the authors.

of them is how we can decide which signal change should be considered large. Authors propose to take such decision on the basis of the distance of sampling points from the reference feature. Considering approach applied in quality engineering we propose to use a value of experimental standard deviation $\mathrm{s}$ as an indicator in this case. Points that are located at a distance higher than $3 \mathrm{~s}$ are the ones where additional measurements should be considered.

Thus, the procedure of searching the points where additional measurements should be per- 
formed can be described as follows:

- performing preliminary measurement,

- calculation of the parameters of the reference feature,

- calculation of the value of the experimental standard deviation s,

- searching for points whose distance from the reference feature is greater than $3 \mathrm{~s}$.

\subsection{Adaptive strategy using helical trace measurement}

In general, this strategy consists of following stages:

1. Preliminary measurement of the workpiece with the use of helical trace strategy.

2. An analysis of measurement data aiming at detection if there are significant local changes of the signal.

3. If significant changes of the signal are detected, then in such area of the surface additional measurements are to be conducted. The additional measurement consists of two measurements of $2 D$ features. The former is the measurement along the generatrix and the latter is the circumferential measurement, which is shown in Fig. 6. It is noteworthy that the trajectories of additional measurements should go across the local extreme value of the profile.

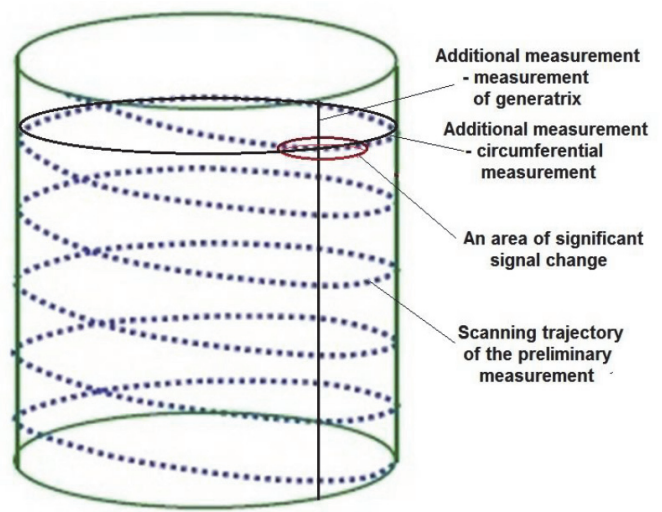

Figure 6: An idea of the adaptive strategy with the use of helical trace measurement

\subsection{Adaptive strategy using generatrix measurements}

In general, this strategy consists of following stages:

1. Preliminary measurement of the workpiece with the use of generatrix measurement.

2. An analysis of measurement data aiming at detection if there are significant local changes of the signal.

3. If significant changes of the signal are detected, then in such area of the surface additional measurements are to be conducted. The additional measurement consists of roundness measurement in the cross-section defined by the local extreme value of the surface. The idea of this strategy is shown in Fig. 7.

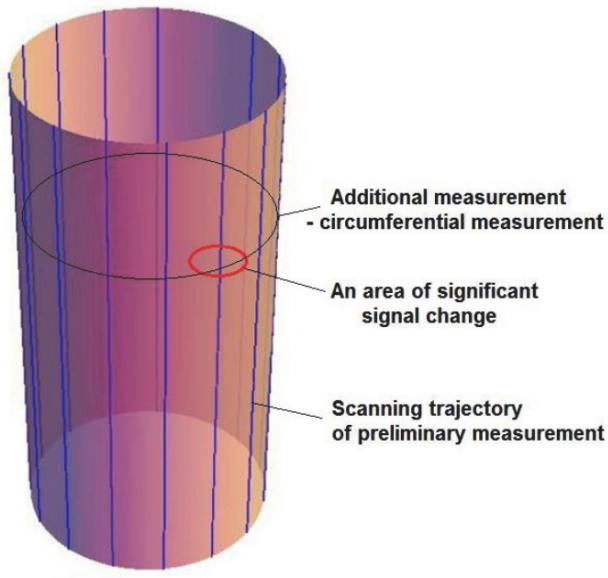

Figure 7: An idea of the adaptive strategy with the use of generatrix measurement.

\subsection{Adaptive strategy using circumferential measurements}

In general, this strategy consists of following stages:

1. Preliminary measurement of the workpiece with the use of circumferential measurement.

2. An analysis of measurement data aiming at detection if there are significant local changes of the signal.

3. If significant changes of the signal are detected, then in such area of the surface additional measurements are to be conducted. The additional measurement consists of straightness measurement of the generatrix that goes across the local extreme value of the surface. The idea of this strategy is shown in Fig. 8.

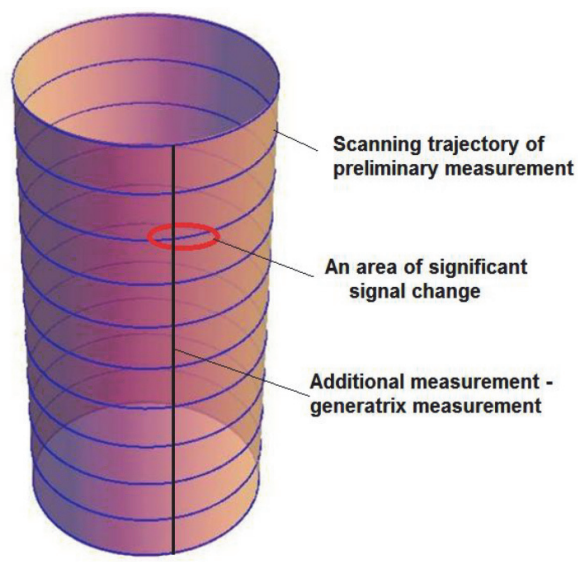

Figure 8: An idea of the adaptive strategy with the use of circumferential measurement.

Authors are going to apply the strategies shown in Figures 6, 7 and 8 to measure such elements as cylinders, spheres, cones, and barrel-shaped workpieces. It will involve solving a number of theoretical 
problems, including developing the mathematical models of the strategy. The developed model should respect such problems as: correct calculation of a reference feature, filtering of measurement data, and non-uniform sampling. On the basis of formulated equations, computer procedures will be developed to allow qualitative (visual) and quantitative (with the use of suitable numerical parameters) evaluation of form deviations of measured workpiece.

\section{Discussion}

Strategies based on predicted model of irregularities and adaptive strategies described in the section 2 are still under investigation and they have not been widely applied under industrial conditions. They have many advantages, however, also severe drawbacks. The strategy based on the predicted model of irregularities allows for accurate sampling only in preselected areas. However, real technological processes are disturbed by many random factors that can contribute to occurring irregularities in areas different from those that were predicted before the process initialization (in particular if the process becomes statistically unregulated). Referring to the problem of adaptive strategies described in Section 2 it should be noted that these are iterative methods, and thus they are quite complex computationally. Moreover, their application with the use of a coordinate measuring machine can be troublesome, as there is a risk of collision of the measuring tip with the surface of the sample [7-9]. The risk results from the fact that sampling points can generally be different for elements whose nominal dimensions are the same. In addition, it is noteworthy that at present time form deviations are usually measured by coordinate measuring machines equipped with scanning probe heads. Such probe heads allow us to obtain very dense sampling in a relatively short time. Thus, methods for whom areas requiring denser sampling are to be calculated become outof-date very quickly. These methods are still relevant if touch-triggering probe heads are used but such type of probe heads is dynamically replaced with scanning ones at present time.

This is the reason why authors propose to apply a novel two-stages adaptive strategy. Authors assume that the adaptive strategy will enable accurate investigation of local irregularities of an observed surface. In addition, assumed philosophy of an adaptive measurement will permit selecting suitable measurement strategy to the surface under investigation. It will make it possible to reduce measurement time along with achieving high accuracy of evaluation of form deviations of the surface. This is why the concept of the adaptive measurement seems to be very interesting.

\section{Conclusions}

Measurements of form deviations of 3D elements can be conducted with the use of various strategies, differing in a number and distribution of sampling points located on an investigated surface. Low number of sampling points or measured sections can lead to the situation that some surface irregularities are not detected by a measuring system. An application of higher density of sampling points, in turn, results in significant lengthening of measurement time, which is undesirable, if one takes into account requirements of modern manufacturing processes.

This is the reason why authors are conducting research work aiming at development of a novel, two-stages adaptive measurement strategy. The proposed adaptive strategy has not been discussed or described in the scientific literature so far. In addition, even leading manufacturers of modern metrological equipment do not offer similar options in the software packages of their measuring instruments. Proposed strategy changes existing approach to measurements. It is assumed that it will permit achieving high measurement accuracy and reduction of measurement time when compared to the strategies that are currently applied. However, it is noteworthy that the strategy will require development of mathematical models of measurement and evaluation of form deviations of rotary elements, which is under investigation at present time. After successful finish of the theoretical part of the work, the authors will verify the concept experimentally. One of the main aims of this study will be the verification of developed software and the exact evaluation of the effect of the new strategy on the reduction of measurement time.

\section{Acknowledgments}

The paper has been elaborated within the framework of the research project entitled "Theoretical and experimental problems of integrated 3D measurements of elements' surfaces", reg. no.: 2015/19/B/ ST8/02643, ID:317012, finances by National Science Centre, Poland. 


\section{References and Notes}

1. Taylor Hobson - Training materials. Available online: URL: http://www.taylorhobsonserviceusa.com/trainingmaterials.html (accessed on 30 November 2017).

2. Adamczak S., Stepien K., Zmarzły P., An analysis of strategies of measurement of 3D rotary elements, Proceedings of the 28th DAAAM International Symposium on Intelligent Manufacturing and Automation, Zadar, Croatia, November 2017.

3. Capello, E., Semeraro, Q. The harmonic fitting method for the assessment of the substitute geometry estimate error. Part I: 2D and 3D theory. Int J Mach Tools Manuf. 2001, vol. 41(8), pp. 1071-1102.

4. Summerhays, K. D. et al. Optimizing discrete point sample patterns and measurement data analysis on internal cylindrical surfaces with systematic form deviations. Precision Engineering 2001, vol. 26, pp. 105-121.

9. Gapiński B., Rucki M. The Roundness Deviation Measurement with CMM, AMUEM 2008 - International Workshop on Advanced Methods for Uncertainty Estimation in Measurement Sardagna, Trento, Italy, 21-22 July 2008, pp. 108-111.

10. Adamczak S., Stępień K.: An analysis of strategies of form deviations' measurements of rotary elements, Mechanik, vol. 2/2018, pp. 113-116

11. Adamczak S., Stępień K.: An analysis of methods of measurement and evaluation of sphericity deviations, Mechanik, vol. 2/2018, pp. 122-125
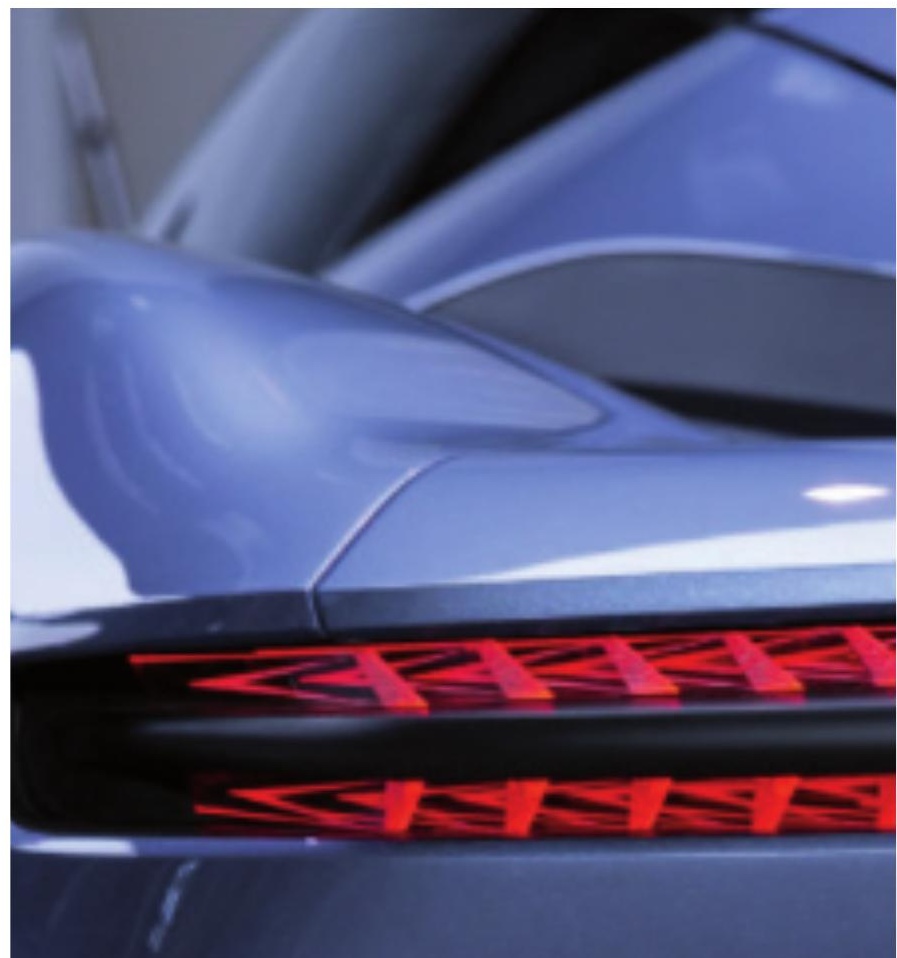